\title{
Stability of internally mixing flow
}

\author{
K MURALIDHAR* and F A KULACKI** \\ *Department of Mechanical Engineering, Indian Institute of Technology, \\ Kanpur 208016 , India \\ **College of Engineering, Colorado State University, Fort Collins, \\ CO 80523, USA
}

MS received 26 October 1987

\begin{abstract}
Hydrodynamic stability of viscous shear flow resulting from the mixing of two parallel streams of fluid along the centre line of a channel is investigated. A linear stability analysis is employed and eigenvalues are extracted using a variational principle combined with a Rayleigh-Ritz procedure. Velocity profiles for the base flow are obtained from a numerical solution of the laminar mixing problem. Results show a sharp drop in critical Reynolds number as the streams start to mix. However, the flow is generally stable at the mean flow Reynolds number investigated here. These results have practical implications for enhancing mixing and heat or mass transfer in thermal systems of small characteristic dimensions, as found in microelectronic equipment packages.
\end{abstract}

Keywords. Hydrodynamic stability; viscous shear flow; Rayleigh-Ritz procedure; laminar mixing problem.

\section{Introduction}

The term "complex flow" is widespread in the study of turbulent flows. Such flows are characterized in general by a behaviour which makes the thin shear layer (TSL) approximation inapplicable. Wall jets, flows with free stream turbulence, flows showing the effects of wall roughness, stratified shear flows and others fall in this category. Complex flows have defied generalization thus far, and require a case-bycase analysis of the flow details. A partial attempt at unification has been attempted recently (Muralidhar 1985) for a class of flows arising from the mixing of two equal parallel streams confined in a channel. The velocity ratio $\mathrm{U}_{2} / \mathrm{U}_{1}$, as seen in figure 1 below, arises as the new parameter of this problem.

Depending on the velocity ratio, distinct regimes of flow begin to appear in the channel. When it is unity, flow development occurs through boundary layers which grow on the walls. When the value is far different from unity, mixing layers along the interface appear over a considerable length of the channel. In turbulent flows, this means that the kinetic energy production is confined either to the wall region or the interface depending on the velocity ratio. For moderate values of $U_{2} / U_{1}$ 
$(=3$, as used here), turbulence production is still predominant in the wall region, and to this extent is boundary layer-like flow. However, the initiation and evolution of turbulence is linked to the inflexion-point instability arising in the interfacial region. In this respect, the flow demonstrates a "mixing-layer" character. The present work provides a linear stability calculation to explore this aspect.

Two layers of a fluid, with different mean velocities, superposed on each other are hydrodynamically unstable in the inviscid limit. The present work involves the calculation of the critical Reynolds number associated with the stability of superposed flows confined in a two-dimensional channel. The presence of constraining walls is included in the framework of viscous shear flows. Close to the entrance of the channel, the boundary layers which grow on the walls are highly stable with the critical Reynolds number being several orders of magnitude higher than the value for fully developed plane Poisseulle flow. The interest here is in understanding the competing influences of the wall (which is stabilizing) and the mixing region near the centre line of the channel. Linear stability theory is employed in the analysis of the perturbed flow. The analysis is completely twodimensional, and the mean flow laminar velocity profiles have been obtained from a finite-difference solution (Muralidhar 1985).

Chen \& Sparrow (1967) have investigated the stability of plane parallel developing flow in a channel, starting from the entrance to the fully developed region of flow. Unbounded shear flows influenced by stratification have been studied by Hazel (1972) at the inviscid-limit. Gage (1971) has provided stability results for stratified shear flows in a channel when the mean velocity profile is taken as Grohne's inflexion-point profile. The idea behind present calculations has been to use realistic mean flow profiles, along with a generalized numerical procedure to solve the eigenvalue problems arising in the stability analysis.

\section{Analysis}

\subsection{Formulation}

Linear stability of viscous flows is governed by the Orr-Sommerfeld equation (Schlichting 1979) given below.

$$
(i / \alpha R e)\left(D^{2}-\alpha^{2}\right)^{2} \phi+(\bar{u}-c)\left(D^{2}-\alpha^{2}\right) \phi+\xi_{y} \phi=0 .
$$

Here, $\phi=\phi(y)$ and is defined as,

$$
\bar{\psi}=\phi(y) \exp [i \alpha(x-c t)]
$$

where $\bar{\psi}$ is the disturbance stream function. The wave number, $\alpha$, is real, and $c$ is the complex wave speed,

$$
c=c_{r}+i c_{i}
$$

The mean flow velocity, $\bar{u}$, and vorticity, $\xi_{y}$, are specified from finite-difference calculations.

\subsection{Boundary conditions}

The disturbance stream function amplitude, $\phi$, satisfies the following conditions on 
the walls (figure 1).

$$
\begin{array}{ll}
y=0, & \phi=\mathrm{D} \phi=0, \\
y=2, & \phi=\mathrm{D} \phi=0 .
\end{array}
$$

Equations (1) and (4) constitute an eigenvalue problem, where $\phi$ is the eigenvector, corresponding to $c$ as an eigenvalue, with $\alpha$ and $\operatorname{Re}$ as parameters. The object is then to look for the smallest $\operatorname{Re}$, for all possible values of $\alpha$, for which $c_{i}$ changes sign from negative to positive.

\subsection{Method of obtaining eigenvalues}

A variational principle has been set up to obtain complex eigenvalues of (1) subject to (4). This principle, along with the subsequent Rayleigh-Ritz procedure is adapted from the work of Lee \& Reynolds (1967). The resulting homogeneous system of algebraic equations constitutes a generalized matrix eigenvalue problem. An $L-Z$ algorithm has been used in the present study to extract the eigenvalues (Kaufman 1975). When the flow is symmetric about the centre line, the trial functions $f_{n}$ used to approximate $\phi$ are given as follows.

$$
\begin{aligned}
f_{n} & =\frac{1}{2}\left[1-(y-1)^{2}\right]^{2}(y-1)^{2 n} \\
n & =0,1, \ldots N .
\end{aligned}
$$

Otherwise,

$$
\begin{aligned}
f_{n} & =\frac{1}{2}\left[1-(y-1)^{2}\right]^{2}\left[(y-1)^{2 n}+(y-1)^{n}\right] \\
n & =0,1, \ldots N .
\end{aligned}
$$

\subsection{Size convergence}

As $N$ increases, the eigenvalue which repeats itself is the desired value of $c$ and furthermore, approaches the exact value. Values of $N$ ranging from five to thirty have been tried, and results for $N$ equal to 25 have been reported.

\subsection{Laminar velocity profiles}

The finite difference methods used to obtain the mean velocity profile in this work have been described fully by Gosman et al (1969). An algebraic system of equations is obtained by integrating the governing equations over a small control volume and using upwind differencing for the nonlinear terms. The entrance boundary condition involves specifying velocity completely, which is the usual Drichlet boundary condition. At the channel exit, suitably located, a gradient boundary condition is used. Wall treatment is standard and is given by Gosman et al (1969). The calculation has been done on a $401 \times 41$ uniform grid, where the $x$-spacing is so chosen that the solution does not strongly depend on the location of the channel exit (Muralidhar 1985).

\subsection{Determination of critical point}

The approach taken in this work involves the following steps. For a prescribed velocity ratio and Reynolds number of base flow, the laminar velocity profiles describing development of flow in the parallel channel are first determined using the finite difference procedure. Assuming that these profiles are physically realizable, 
they are subsequently destabilized by small disturbances. Instability is indicated by the positive sign of $c_{i}$. Stability of velocity profiles at various positions along the channel length have been studied. For a given location, the wave number $\alpha$ is kept fixed and $\mathrm{Re}$ in the Orr-Sommerfeld equation is increased till $c_{i}$ becomes slightly larger than zero. A set of Reynolds numbers can then be obtained by varying $\alpha$, all of which make $c_{i}$ just positive. The minimum in this set is the critical Reynolds number for the location under consideration. If this value is smaller than the Reynolds number of base flow, the profile is taken to undergo transition to turbulence.

\section{Results}

For fully developed plane Poisseulle flow, the critical Reynolds number calculated here is $6150(10)$, based on the maximum velocity in the channel and its half-width. The quantity 10 in brackets gives the extent of uncertainty in the calculated value, and arises from not setting $c_{i}$ as exactly zero. Values ranging from 5400 to 5800 have been obtained by Chen $\&$ Sparrow (1967), using a variety of other numerical techniques. Table 1 shows size convergence properties observed with the scheme used in this work. Similar results have been obtained by Lee \& Reynolds (1967). Typically close to instability, size convergence is poor even with a very large number of trial functions. Further, it is destroyed for large values of critical Reynolds number, typically $\alpha \operatorname{Re}>50,000$ (Glandsdorff \& Prigogine 1971). This problem can however be handled via ortho-normalization techniques described elsewhere (Muralidhar \& Kulacki 1987).

The mean flow is obtained by solving an elliptic problem, subject to specified data on the inlet boundary and a gradient condition at the exit, using a finite difference scheme. Accordingly, the absolute error in solution increases downstream, being zero at the entrance. This is a consequence of using the Dirichlet condition on part of the flow domain. The stability calculation overlaps with this region of low error in mean velocity profile. At distances farther away from the inlet, the inflexion point profiles get rounded off, and become quite stable. The stability calculation is inapplicable here since $\alpha \mathrm{Re}$ is definitely larger than 50,000 . Hence, it is fortuitous that the region of low finite differencing error, the region where the Rayleigh-Ritz procedure of calculating eigenvalues is valid and the region where the flow is seriously destabilized, all overlap.

Table 1. Size convergence in fully developed flow.

\begin{tabular}{|c|c|c|c|c|}
\hline & $\alpha=1.0$ & $\operatorname{Re}=100$ & $\alpha=1.0$ & $\operatorname{Re}=8000$ \\
\hline$N$ & $c_{r}$ & $c_{i}$ & $c_{\mathrm{r}}$ & $c_{i}$ \\
\hline 5 & 0.4773 & $-0 \cdot 16143$ & $0 \cdot 2655$ & 0.02958 \\
\hline 8 & 0.4772 & $-0 \cdot 161406$ & $0 \cdot 24123$ & 0.01161 \\
\hline 10 & 0.4772 & -0.16139 & 0.2295 & 0.01109 \\
\hline 15 & & & 0.2446 & 0.00632 \\
\hline 20 & 0.47716 & $-0 \cdot 161524$ & 0.2588 & 0.000917 \\
\hline 25 & & & 0.2444 & 0.00414 \\
\hline 30 & & & 0.2487 & 0.00786 \\
\hline
\end{tabular}




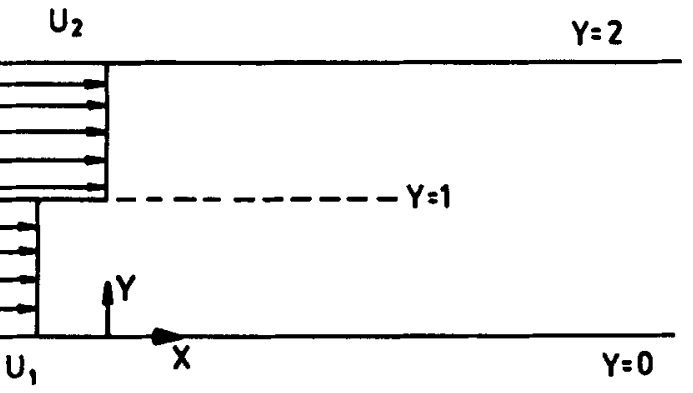

Figure 1. Flow configuration and coordinate system.

Figure 1 shows the physical problem being considered, the velocity ratio $U_{2} / U_{1}$ being 3. Reynolds number based on the mean velocity in the channel is 500 . Figure 2 shows the finite difference solution for this case. Figure 3 is the stability envelope for the profile at $x=0.54$. The region inside this curve represents unstable states which cannot be accessed by the flow. The region outside is stable, within the framework of linear stability theory. The critical Reynolds number at $x=0.54$ is calculated as 960 (2). Figure 4 shows the variation of critical Reynolds number with distance from inlet, for a base $\operatorname{Re}$ of 500 . Figure 5 shows the same for a base $\operatorname{Re}$ of 1000. The lowest critical Reynolds number in figure 5 can be seen to be 1200 . In either case, the critical Reynolds number is above the base flow Reynolds number. The appropriate conclusion is that the flow is stable, in each case. However, in view of the closeness of the critical and the base flow Reynolds numbers, a possible suboptimal choice of trial functions and the possibility of nonlinear effects, stability cannot be unconditionally ensured. The influence of either increasing the velocity ratio or the base $\mathrm{Re}$ is destabilizing, but separation of flow on the high velocity side could inhibit transition to turbulence.

Some conclusions can be drawn from the figures referred to earlier. If the flow at inlet had been parallel (that is, a velocity ratio of unity), then the critical Reynolds numbers close to the inlet would have been several orders of magnitude greater than the value of 6000 , quoted for fully developed plane Poisseulle flow. For a velocity ratio of 3 , this value is seen to be around 1000 , for both Re equal to 500

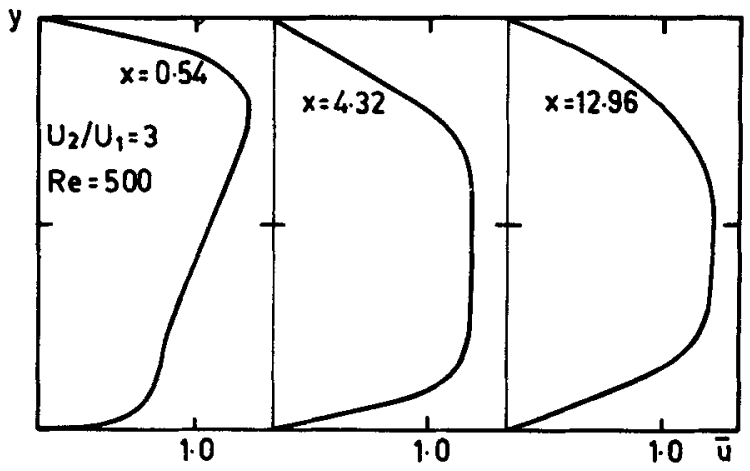

Figure 2. Finite difference solution for mean flow at three locations along channel length. 


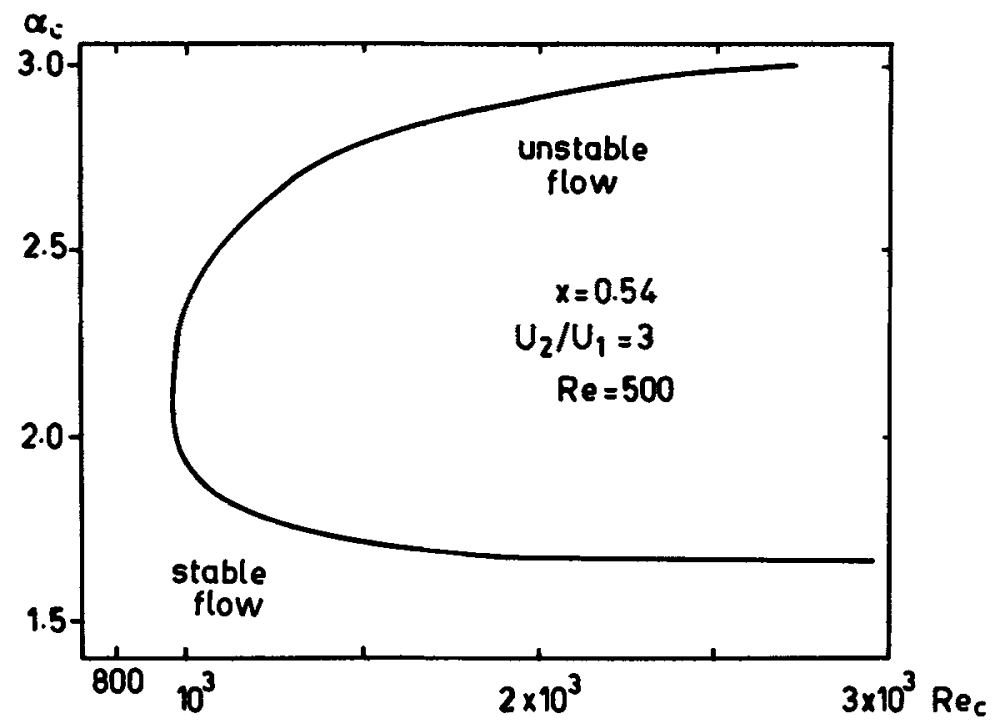

Figure 3. Stability envelope of mixing flow.

and 1000 . This drastic reduction in $\operatorname{Re}_{c}$ reveals that the flow is highly prone to becoming turbulent in the entranice region. While the stability of flow at a velocity ratio of unity can be attributed to the thinness of boundary layers, the destabilizing influence for a velocity ratio of three is due to the formation of inflexion-point profiles during flow development. For both base flow Reynolds numbers studied here, the extent to which the destabilizing influence is present is about one channel height. Beyond this length, the critical Reynolds number shows a sharp increase. It can then be concluded that the flow here reverts to a boundary-layer character. There is one difference in the behaviour of velocity profiles, as the base Reynolds number is increased. This relates to the critical Reynolds number in the depressed

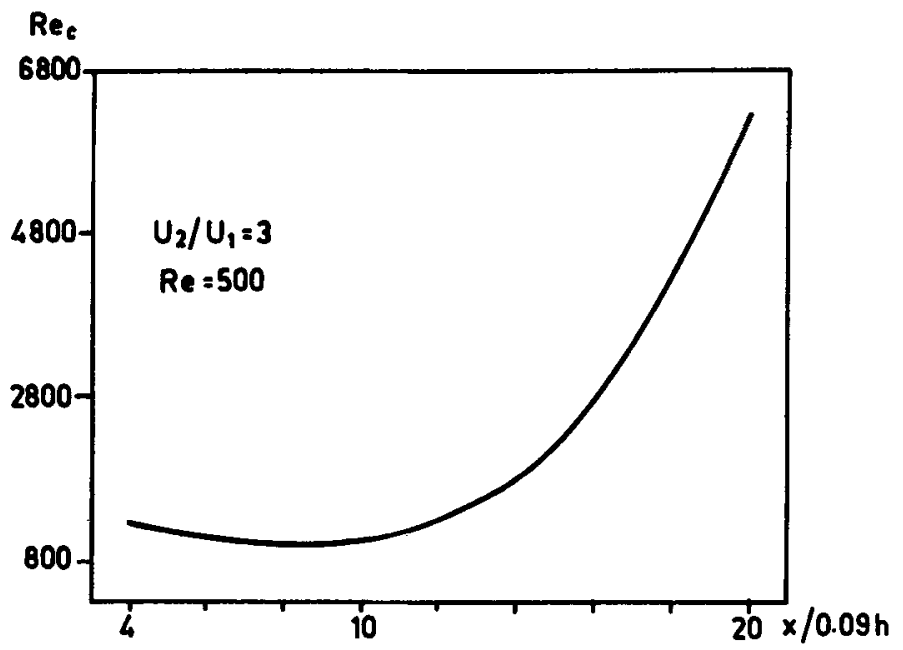

Figure 4. Critical Reynolds number as a function of distance from channel inlet. 


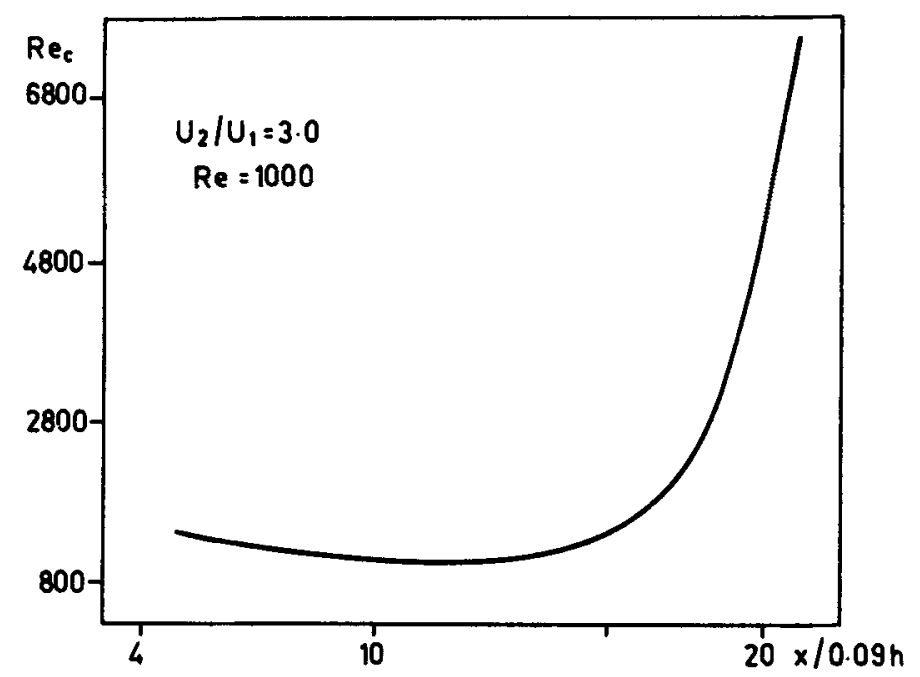

Figare 5. Critical Reynolds number as a function of distance from channel inlet.

region itself, where the inflexion point occurs. At a base $\operatorname{Re}$ of 1000 , the depression in the critical Re-curve exists over a longer distance, in comparison to the case of the base $\operatorname{Re}$ of 500 . The pressure drop in the channel and the transport coefficients depend primarily on whether the flow is laminar or turbulent. For this reason, more studies of the type reported in this work are required to determine the points of transition as a function of Reynolds number and velocity ratio.

The present results have practical implications for enhancement of heat or mass transfer in mixing flows confined in systems of small characteristic dimensions. Examples are to be found in compact heat exchanger design and cooling of electronic equipment. With a stable mixing flow, turbulence promoters would most certainly be needed either upstream in the inlet flow or at the commencement of mixing.

\section{List of symbols}

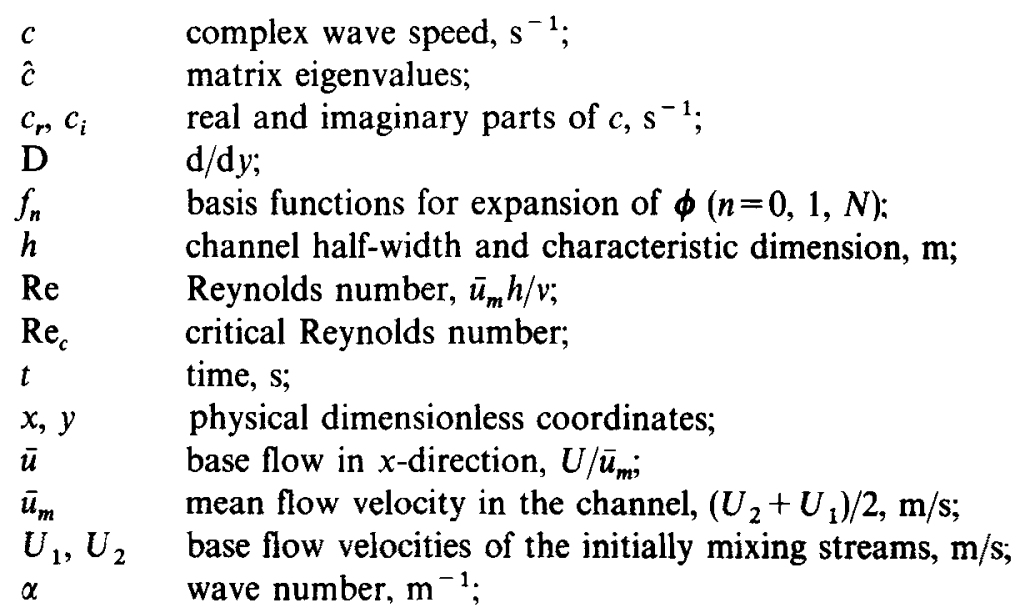




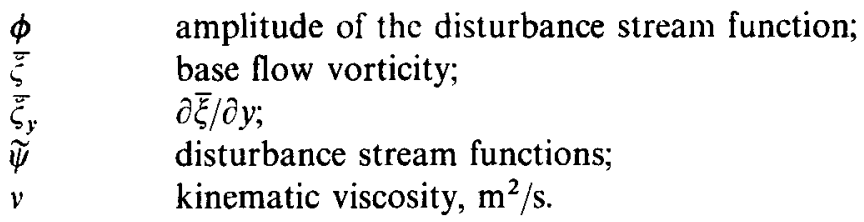

\section{References}

Chen T S, Sparrow E M 1967 J. Fluid Mech. 30: 209-224

Gage K S 1971 J. Fluid Mech. 47: 1-20

Glandsdorff P, Prigogine I 1971 Thermodynamic theory of structure, stability and fluctuations (New York: Wiley-Interscience)

Gosman A D, Pun W M, Runchal A K, Spalding D B, Wolfschtein M 1969 Heat and mass transfer in recirculating flows (New York: Academic Press)

Hazel P 1972 J. Fluid Mech. 51: 39-61

Kaufman L C 1975 ACM Trans. Math. Software 1: 271-281

Lee L H, Reynolds W C 1967 Q. J. Appl. Math. 20: 1-22

Muralidhar K 1985 Buoyancy effect on mixing flow, Doctoral Dissertation, University of Delaware, Newark

Muralidhar K, Kulacki F A 1987 Int. J. Heat Fluid Flow 8: 228-233

Schlichting H 1979 Boundary-layer theory (New York: McGraw Hill) 